Article

\title{
Battery Remaining Useful Life Prediction with Inheritance Particle Filtering
}

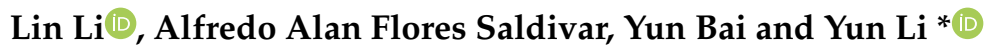 \\ Industry 4.0 Artificial Intelligence Laboratory, School of Computer Science and Technology, \\ Dongguan University of Technology, Dongguan 523808, China \\ * Correspondence: Yun.Li@ieee.org
}

Received: 12 May 2019; Accepted: 16 July 2019; Published: 19 July 2019

check for updates

\begin{abstract}
Accurately forecasting a battery's remaining useful life (RUL) plays an important role in the prognostics and health management of rechargeable batteries. An effective forecast is reported using a particle filter $(\mathrm{PF})$, but it currently suffers from particle degeneracy and impoverishment deficiencies in RUL evaluations. In this paper, an inheritance PF is developed to predict lithium-ion battery RUL for the first time. A battery degradation model is first mapped onto a PF problem using the genetic algorithm (GA) framework. Then, a Lamarckian inheritance operator is designed to improve the light-weight particles by heavy-weight ones and thus to tackle particle degeneracy. In addition, the inheritance mechanism retains certain existing information to tackle particle impoverishment. The performance of the inheritance PF is compared with an elitism GA-based PF. The former has fewer tuning parameters than the latter and is less sensitive to tuning parameters. Both PFs are applied to the prediction of lithium-ion battery RUL, which is validated using capacity degradation data from the NASA Ames Research Center. The experimental results show that the inheritance PF method offers improved RUL prediction and wider applications. Further improvement is obtained with one-step ahead prediction when the charging and discharging cycles move along.
\end{abstract}

Keywords: lithium-ion battery; battery remaining useful life; particle filter; evolutionary computation

\section{Introduction}

Optimized lithium-ion batteries play an important role in daily applications, from mobile phones, through microgrids, to satellites [1,2]. Despite the fact that they offer many advantages over other types of batteries, such as a low weight, high power density, and long lifespan [3], their performance degrades with repeated charging and discharging. Hence, their degradation identification, state estimation and prediction, and maintenance optimization have become a focus of attention in engineering research [4]. To optimize the energy deployment of a battery, avoid its premature failure, and improve its reliability and durability, it is important to monitor its degradation process accurately. This involves evaluating the state of health $(\mathrm{SOH})[5]$ and predicting the state of charging $(\mathrm{SOC})$ and remaining useful life (RUL) [6] in a battery management system (BMS) [7], which can be used for automated and optimized scheduling of maintenance actions that, in turn, ensure safe operation and use of lithium-ion batteries. Capacity is a direct indicator of $\mathrm{SOH}$, where the end of life (EOL) is defined as the state in which the capacity reaches $70-80 \%$ of the nominal value [8] at the same SOC and operating conditions.

In recent years, a number of RUL prediction methods have been developed. For instance, neural networks [9] and relevance vector machines (RVMs) [10] have been utilized to learn the battery $\mathrm{SOH}$, using test data to estimate the parameters of a battery degradation model. However, these methods are dependent on the training datasets and their models lose accuracy under complex conditions [11]. A more adaptive method is a parameter estimation approach, using a filter such as the extended Kalman filter (EKF) [12], unscented Kalman filter [13], or their improved versions [14-16], which 
can adjust the model parameters to track battery degradation. Despite this improvement, however, these Kalman filters cannot be well adjusted since battery life generally demonstrates a nonlinear and non-Gaussian property.

To realize further improvements, the particle filter (PF) widely adopted in sequential importance resampling (SIR) $[17,18]$ has been used to provide better estimation and prediction for battery SOH [19]. For example, a PF framework for prognostics of lithium-ion battery systems is presented in [20]. However, it is known that the estimation error for PF can be large for several instances during the model parameter training stage due to the degeneracy and impoverishment of particles [21]. To solve these problems, improved particle filtering methods have been developed recently. These have led to improved RUL estimation for lithium-ion batteries in evaluating $\mathrm{SOH}$. For instance, an empirical degradation model and unscented particle filtering have been applied to predicting the RUL [22]. Predicting the end-of-discharge of a lithium-ion battery has also been reported by combining a PF and an artificial neural network [23]. In the state space, capacity degradation has been estimated using a spherical cubature particle filter [24]. Markov-chain-based Monte Carlo simulation has also been shown to be effective with an improved unscented PF for sample impoverishment in lithium-ion battery RUL prediction [25].

The review in [6] has concluded that, because the battery modeling methods currently reported in the literature are not accurate enough and impose several restrictions to continually update the models, it is necessary to perform more research in this field. Recently, further improvements in overcoming the particle diversity deficiency have been reported [26], as well as the use of an improved unscented particle filter in optimizing combination resampling for RUL prediction [27] and the use of a heuristic Kalman filter [28]. Despite the efforts undertaken so far, however, the issues of particle degradation and particle impoverishment remain challenging for RUL estimation. The work reported in $[27,28]$ has particularly motivated the work reported in this paper. From the review in [6], it is clear that adaptive filter-based algorithms are more suitable for electric vehicle (EV) applications, and algorithms based on artificial intelligence (AI) are not suitable for this application due to its intensive computing and learning requirements. For solving the optimization problem in filter-based techniques, however, the trend is to use AI-based techniques owing to their simplicity, flexibility, derivation-free mechanism, and local optima avoidance [6].

Hence, we propose an AI-based inheritance particle filtering approach to the prediction of lithium-ion battery RUL with high accuracy, using the genetic algorithm (GA) framework. A Lamarckian inheritance operator is developed to reduce particle degradation, enhance particle diversity and simplify the filtering process. Thus, the optimized particle will tend to cluster around the high likelihood region. Battery capacity degradation data provided by NASA Ames Research Center will be used to validate the proposed prediction approach and to compare it with the GA-based PF approach.

The rest of the paper is organized as follows. In Section 2, the empirical degradation model of a lithium-ion battery solved in this paper is described. In Section 3, brief preliminaries regarding the standard PF are presented and the inheritance PF algorithm is developed for building a prediction model of the RUL. In Section 4, we first use one experimental example to demonstrate the effectiveness of the inheritance PF. Then, the results of the standard PF and inheritance PF for RUL prediction are compared and analyzed, using real lithium-ion battery data extracted from the NASA Ames Research Center database. Finally, in Section 5, conclusions are summarized, and future work highlighted.

\section{Empirical Degradation Model of Lithium-Ion Battery RUL}

A lithium-ion battery and its degradation involve a complex electrochemical process. In particular, self-charging during charge and discharge cycles makes it difficult to establish a suitable first-principles-based clear- or grey-box model [29] to characterize the overall degradation process. Hence, a black-box model such as the empirical model of the capacity degradation from He et al. [30] 
has been adopted. For example, RUL prediction of lithium-ion battery capacity is approximated by a double-exponential degradation black-box model as [30]:

$$
Q=a \cdot \exp (b \cdot k)+c \cdot \exp (d \cdot k)
$$

where $a, b, c$ and $d$ are the parameters of the double-exponential function, the amplitudes of which, $a$ and $c$, are related to the internal impedance of the battery and the degradation rates of which, $b$ and $d$ are related to aging; $k$ is the number of charging cycles, and $Q$ the remaining capacity of the battery.

Here, the objective of modeling and hence predicting the RUL is to estimate these model parameters. They can be estimated as transitional states and measurements of a stochastic dynamic system [30]:

$$
\begin{aligned}
& \mathbf{x}_{k}=\left[a_{k} b_{k} c_{k} d_{k} \mu \sigma\right]^{\mathrm{T}}, \\
& \left\{\begin{array}{ll}
a_{k}=a_{k-1}+w_{a} & w_{a} \sim N\left(\mu, \sigma_{a}\right) \\
b_{k}=b_{k-1}+w_{b} & w_{b} \sim N\left(\mu, \sigma_{b}\right) \\
c_{k}=c_{k-1}+w_{c} & w_{c} \sim N\left(\mu, \sigma_{c}\right) \\
d_{k}=d_{k-1}+w_{d} & w_{d} \sim N\left(\mu, \sigma_{d}\right)
\end{array},\right. \\
& Q_{k}=a_{k} \cdot \exp \left(b_{k} \cdot k\right)+c_{k} \cdot \exp \left(d_{k} \cdot k\right)+n_{k} \text {, }
\end{aligned}
$$

where $Q_{k}$ is the capacity of the battery at cycle index $k$ and $N(\mu, \sigma)$ the Gaussian noise with mean $\mu$ and standard deviation (SD) $\sigma$, which could be different for different parameters, but are usually constant and hence not expressed as state variables to estimate. To determine the probabilistic characteristics of the estimation, the state variables are estimated conditionally based on observation information, which can be achieved via particle filtering.

\section{Inheritance Particle Filtering Approach to RUL Prediction}

Here, we propose an inheritance particle filtering approach to predicting battery RUL, using the GA framework to develop an inheritance-based Lamarckian particle filter (LPF). A Lamarckian operator is designed in the GA context to modify the light-weight particles by heavy-weight ones and to retain the information of light-weight particles. This is to improve from the standard PF the diversity of resampled particles, to mitigate the particle degeneracy and impoverishment problem. In the following, the standard PF will be first outlined, followed by the LPF algorithm.

\subsection{Particle Filter Algorithm}

A particle filter is also known as a sequential Monte Carlo method in statistics. It combines Bayesian learning with importance sampling to estimate a posterior probability density function with a set of weighted particles representing sampled values from an unknown state space. Particle filtering has thus found wide applications in stochastic processes of given noisy and/or partial observations, such as the prediction of a battery's remaining lifetime [22].

Many dynamic processes can be described by a state space model [31], including the state transition and measurement equations written as:

$$
\begin{gathered}
\mathbf{x}_{k}=f\left(\mathbf{x}_{k-1}, \mathbf{u}_{k-1}\right), \\
\mathbf{z}_{k}=h\left(\mathbf{x}_{k}, \mathbf{v}_{k-1}\right),
\end{gathered}
$$

where $\mathbf{x}_{k}$ and $\mathbf{z}_{k}$ are the state variables and observations at time $k$, respectively; $\mathbf{x}_{k} \in \mathbf{R}^{n_{\mathbf{x}}}, \mathbf{z}_{k} \in \mathbf{R}^{n_{\mathbf{z}}}, n_{\mathbf{x}}$ and $n_{\mathbf{z}}$ are the dimensions of $\mathbf{x}_{k}$ and $\mathbf{z}_{k}$, respectively; $f(\cdot)$ and $h(\cdot)$ are the known system and observation functions, respectively; $\mathbf{u}_{k-1} \in \mathbf{R}^{n_{\mathbf{x}}}$ and $\mathbf{v}_{k} \in \mathbf{R}^{n_{\mathbf{z}}}$ are the system and observation noises, respectively, which are both random in terms of known distributions and $\mathbf{v}_{k}$ likely independent of $\mathbf{u}_{k-1}$; and $\mathbf{u}_{k-1}$ and $\mathbf{v}_{k-1}$ are independent of the past and current states. 
A standard PF estimates the posterior distribution of the states using an observation measurement

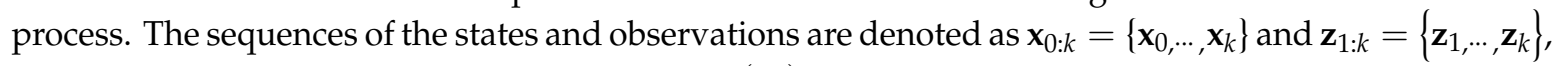
respectively. The initial distribution of states $p\left(\mathbf{x}_{0}\right)$ is known a priori. A PF consists of a mass of $N$ particles $\mathbf{x}_{k}^{i}(i=1, \cdots, N)$, where the number of particles, $N$, remains a constant at each time step $k$. Particle filtering is an approximation, but it can become more accurate with enough particles.

The objective of the aforementioned particle filtering is to estimate the posterior probability density $p\left(\mathbf{x}_{0: k} \mid \mathbf{z}_{1: k}\right)$ using a collection of particles $\left\{\mathbf{x}_{k}^{i}\right\}_{i=1}^{N}$ with associated weight vector $\left\{w_{k}^{i}\right\}_{i=1}^{N}$, based on all available measurements $\mathbf{z}_{1: k}$. Hence, the posterior probability density $p\left(\mathbf{x}_{\mathrm{k}} \mid \mathbf{z}_{1: \mathrm{k}}\right)$ can be written as [31]:

$$
p\left(\mathbf{x}_{k} \mid \mathbf{z}_{1: k}\right) \approx \sum_{i=1}^{N} \widetilde{w}_{k}^{i} \delta\left(\mathbf{x}_{k}-\mathbf{x}_{k}^{i}\right)
$$

where $\delta(\cdot)$ is the Dirac delta function.

Actually, it is difficult to obtain the ideal particle distribution directly from the posterior probability density distribution. Therefore, an "importance sampling" technique is used to sample the particles. The "importance distribution" in the filtering process is given by [31]:

$$
q\left(\mathbf{x}_{0: k} \mid \mathbf{z}_{1: k}\right)=q\left(\mathbf{x}_{0: k-1} \mid \mathbf{z}_{1: k-1}\right) q\left(\mathbf{x}_{k} \mid \mathbf{x}_{0: k-1}, \mathbf{z}_{1: k}\right)
$$

In the PF, a resampling procedure is used to solve the particle degeneracy problem. It is to eliminate samples with a low importance weight and retain those with a high importance weight. Following this resampling process, the weights are estimated as $w_{k}^{i}=1 / N$, and the posterior density $p\left(\mathbf{x}_{k} \mid \mathbf{z}_{1: k}\right)$ is approximated by [31]:

$$
p\left(\mathbf{x}_{k} \mid \mathbf{z}_{1: k}\right) \approx \frac{1}{N} \sum_{i=1}^{N} \delta\left(\mathbf{x}_{k}-\mathbf{x}_{k}^{i}\right)
$$

While the resampling procedure of the PF reduces the particle degeneracy efficiently, particle impoverishment remains to be solved, as the low-weight particles are eliminated, whereas offspring particles share few distinct values. Therefore, the following section develops inheritance particle filtering to tackle this problem.

\subsection{Lamarckian Particle Filter}

\subsubsection{Genetic Algorithm Framework for Particle Filtering}

A GA solves a significant number of complex optimization problems, including the PF problem. The principle of a GA PF is based on Darwin's theory of natural evolution through a "survival-of-the-fittest" selection. Several candidate PF solutions to the PF problem are drawn from its potential solution space. These solutions are optimized by mimicking a natural evolutionary process, through selection, crossover and mutation operations. One example of work using the GA mechanism to construct a PF for improved estimation performance is given in [31]. However, deficiencies such as a low convergence speed, weak local search ability, and premature convergence are not overcome.

\subsubsection{Lamarckian Particle Filter}

Compared with the working mechanism of a standard GA or an elitism GA, the Lamarckian mechanism of evolution is based on the theory that specific characteristics of an organism are directly inheritable by its offspring and survives the given environment [32,33]. In evolutionary algorithms, it has been shown that Lamarckian inheritance is an effective way to improve machine learning and convergence characteristics [34].

Here, in place of the conventional selection and crossover operators of a GA, an overwriting operator is implemented in the LPF on Lamarckian inheritance. This operator ensures that fitter organisms are given the priority directly to pass on their characteristics to their offspring in evolution. Suppose that each of the $N$ particles at time $k, \mathbf{x}_{k^{\prime}}^{i}$ consists of six parameters of the RUL model as 
shown in Equation (2). Each parameter is termed a "phenotype" of "genes". For simplicity of illustration, we assume that each parameter is represented by one gene. A complete set of the genes representing the parameter set is termed a "chromosome". Consider two chromosomes at time $k$, $\mathbf{x}_{k}^{i_{1}}=A=\left(A_{1}, A_{2}, A_{3}, A_{4}, A_{5}, A_{6}\right)$ and $\mathbf{x}_{k}^{i_{2}}=B=\left(B_{1}, B_{2}, B_{3}, B_{4}, B_{5}, B_{6}\right)$, where $A$ is better than $B$, i.e., fitness $(A)>$ fitness $(B)$.

In an LPF, for chromosomes $A$ and $B$ to produce offspring, a certain percentage of the genes of chromosome $B$ will be overwritten by corresponding genes of chromosome $A$, forming chromosome $B^{\prime}$ as a new candidate PF. The percentage of the overwriting genes transferred over to $B^{\prime}$ is termed the Lamarckian probability, which is denoted $p_{t}$ and is calculated as:

$$
p_{t}=\left(\frac{\text { fitness }(A)}{\text { fitness }(B)+\text { fitness }(A)}\right) \%,
$$

The numbers of genes transferred, $n_{\mathrm{t}}$, is calculated by:

$$
n_{\mathrm{t}}=n_{\mathbf{x}} \cdot p_{\mathrm{t}}
$$

where $n_{\mathbf{x}}$ is the number of parameters (or genes in this simplified example) or the number of dimensions of a particle in the PF. This overwriting operation is depicted in Figure 1.

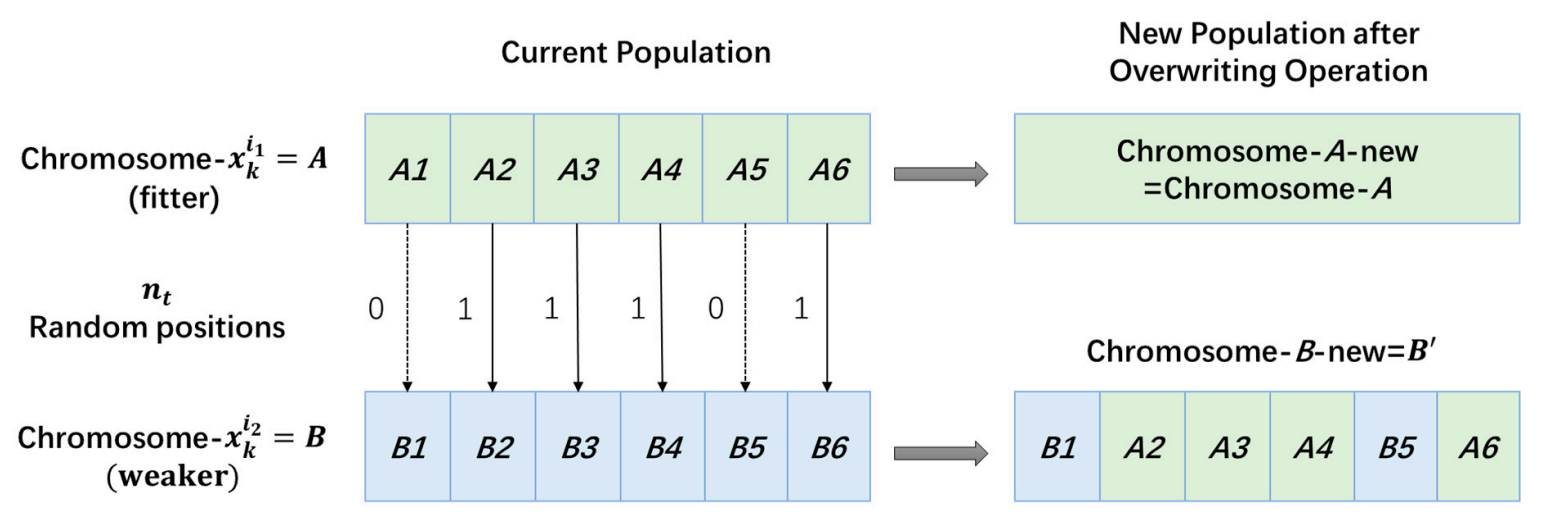

Figure 1. Lamarckian particle filter (LPF) A and LPF B forming offspring B' via overwriting operation.

Hence, the number of genes passed on at crossover depends on the relative fitness of the two parental chromosomes. In this way, even if the fitness of chromosome $B$ is too low, it will retain a small portion of its genes instead of being unselected as in a GA, thereby maintaining a degree of diversity in the gene pool and thus mitigating the particle impoverishment problem.

Further, the overwriting operation saves the mutation operation of a GA. With elitism, the overwriting operations will retain the best characteristics in the PF, while some lightweight particles are still present in the population for particle diversity potentially useful for future explorations.

\subsubsection{Implementation of the LPF Algorithm}

The steps of the LPF for RUL are given below.

Step 1: Initialization

Generate an initialization particle set $\left\{\mathbf{x}_{0}^{i}\right\}, i=1,2, \cdots, N$, by drawing from an assumed or a priori probability density $p\left(\mathbf{x}_{0}\right)$. In the population, each particle or chromosome can be encoded in binary if necessary or if the number of parameters is relatively small, to explore finer estimation. 
Step 2: Importance Sampling to Form an Initial Population

Sample $N$ particles with weights $\left\{\mathbf{x}_{k^{\prime}}^{i}, w_{k}^{i}\right\}_{i=1}^{N}$ using the transition density $q\left(\mathbf{x}_{k}^{i} \mid \mathbf{x}_{k-1}^{i}, \mathbf{z}_{k}\right)=p\left(\mathbf{x}_{k}^{i} \mid \mathbf{x}_{k-1}^{i}\right)$ as the importance density to estimate the weight of each particle:

$$
\widetilde{w}_{k}^{i} \propto \widetilde{w}_{k-1}^{i} \frac{p\left(\mathbf{z}_{k} \mid \mathbf{X}_{k}^{i}\right) p\left(\mathbf{X}_{k}^{i} \mid\left(\mathbf{X}_{k-1}^{i}\right)\right.}{q\left(\mathbf{X}_{k}^{i} \mid\left(\mathbf{X}_{k-1}^{i}, \mathbf{Z}_{k}\right)\right.}
$$

for normalization to

$$
w_{k}^{i}=\widetilde{w}_{k}^{i} / \sum_{i=1}^{N} \widetilde{w}_{k^{\prime}}^{i}
$$

The importance sampling particle set $\left\{\mathbf{x}_{k}^{i}\right\}_{i=1}^{N}$ at $k=0$ forms the initial population of the PF. The size of the population means the number of particles, which remains as $N$, constant to $k$ in the filtering process.

\section{Step 3: Overwriting Operation}

First, the overwriting chromosome is chosen according to the given inheritance probability. For instance, suppose that particles $i_{1}$ and $i_{2}$ are chosen with $w_{k}^{i_{1}}>w_{k}^{i_{2}}$. Then, the probability of each gene in $i_{1}$ to overwrite its corresponding gene in $i_{2}$ is calculated as:

$$
p_{t}=\left(\frac{w_{k}^{i_{1}}}{w_{k}^{i_{1}}+w_{k}^{i_{2}}}\right) \%
$$

The numbers of genes transferred, $n_{t}$, is calculated by (11).

In this way, the lower-weight particle is overwritten in part by the higher-weight one with favorable genes, like gene editing in biochemistry. If the weight of one selected particle is zero, overwriting will not be performed. Note that the overwriting operation will automatically retain the elitist particle, as depicted in Figure 1. Hence, the LPF is always an elitism algorithm, as there is no need to use a mutation operation in an LPF.

Step 4: Evolution Until Termination

The particle set evolves generation by generation until the process terminates when a fixed number of iterations have been reached or no meaningful improvements may be obtained.

Step 5: Outputting the State Estimates

Then, the particle phenotypes are decoded back to real numbers of the optimal particles with equal weights $\left\{\tilde{x}_{k}^{i}, 1 / N\right\}_{i=1}^{N}$. By Equation (9), the system state is thus estimated as:

$$
\mathbf{x}_{k}=\frac{1}{N} \sum_{i=1}^{N} \tilde{\mathbf{x}}_{k}^{i}
$$

\subsubsection{State Updating and Prediction in the LPF Algorithm}

The accurate estimation of battery capacity not only depends on a degradation model, but also on its parameters. According to [30], using the LPF, the estimated capacity for each particle at cycle $k$ is given by:

$$
Q_{k}^{i}=a_{k}^{i} \cdot \exp \left(b_{k}^{i} \cdot k\right)+c_{k}^{i} \cdot \exp \left(d_{k}^{i} \cdot k\right)
$$

and hence the capacity is estimated as:

$$
Q_{k}=\sum_{i=1}^{N} w_{k}^{i} \cdot Q_{k^{\prime}}^{i}
$$


An $L$-step-ahead prediction at cycle $k$ with present observations can be estimated as:

$$
Q_{k+L}^{i}=a_{k}^{i} \cdot \exp \left(b_{k}^{i} \cdot(k+L)\right)+c_{k}^{i} \cdot \exp \left(d_{k}^{i} \cdot(k+L)\right),
$$

The estimated posterior PDF can be predicted at each trajectory with weights:

$$
P\left(Q_{k+L} \mid Q_{0: k}\right) \approx \sum_{i=1}^{N} w_{k}^{i} \delta\left(Q_{k+L}-Q_{k+L}^{i}\right),
$$

Hence, the expectation of the $L$-step-ahead prediction yields:

$$
\bar{Q}_{k+L}=\sum_{i=1}^{N} w_{k}^{i} Q_{k+L^{\prime}}^{i}
$$

As the failure threshold is $70 \%$ of the rated capacity, the RUL estimation $L_{k}^{i}$ of the $i$ th trajectory is obtained by solving the equation:

$$
a_{k}^{i} \exp \left(b_{k}^{i}\left(k+L_{k}^{i}\right)\right)+c_{k}^{i} \exp \left(d_{k}^{i}\left(k+L_{k}^{i}\right)\right)=0.7 Q_{\text {rated }}
$$

Then, the distribution of RUL is estimated as:

$$
P\left(L_{k} \mid Q_{0: k}\right) \approx \sum_{i=1}^{N} w_{k}^{i} \delta\left(L_{k}-L_{k}^{i}\right),
$$

The expectation of the RUL at the cycle $k$ is thus predicted as:

$$
\bar{L}_{k}=\sum_{i=1}^{N} w_{k}^{i} L_{k^{\prime}}^{i}
$$

\section{Experiments and Validation}

Here, the performances of LPF is demonstrated in a single-dimensional (1D) system against the PF that is based on genetic algorithm with elitism (GAe), which is termed GAePF. The LPF is then compared with the standard PF for lithium-ion RUL prediction.

\subsection{Single-Dimension Experiment}

A nonlinear $1 D$ experiment widely used in PF testing is given by [35]:

$$
\begin{gathered}
\mathbf{x}_{k}=1+\sin (\alpha \pi k)+\varnothing_{1} \mathbf{x}_{k-1}+\mathbf{v}_{k}, \\
\mathbf{z}_{k}= \begin{cases}\varnothing_{2} \mathbf{x}_{k}^{2}+\mathbf{r}_{k} & k \leq 30 \\
\varnothing_{3} \mathbf{x}_{k}-2+\mathbf{r}_{k} & k>30\end{cases}
\end{gathered}
$$

where $\mathbf{v}_{k}$ is a random variable modeling the process noise in $\operatorname{Gamma}(3,2) ; \alpha=4 \times 10^{-2}, \varnothing_{1}=\varnothing_{3}=0.5$, and $\varnothing_{2}=0.2$ are scalar parameters in the output model; and the observation noise is $\mathbf{r}_{k}$, drawn from a Gaussian distribution $N(0,0.00001)$. Different filters are used to estimate the state $\mathbf{x}_{k}$ for $k=1,2, \ldots, T$, where the total observation time is $T=70$. The maximum number of generations is $G=20$. In the $\mathrm{LPF}$, the inheritance probability is $\rho=0.5$. In the GAePF, the crossover probability and the mutation probability are 0.5 and 0.1 , respectively. All of the PFs have $N=100$ particles. The experiment was carried out on Monte Carlo simulations with $M=200$.

To evaluate the performance of LPF and GAePF, three root-mean-squared errors are used:

$$
\begin{gathered}
\text { rmse }=\sqrt{\frac{1}{T} \sum_{k=1}^{T}\left(\mathbf{x}_{k}-\hat{\mathbf{x}}_{k}\right)^{2}}, \\
\overline{r m s e}=\sqrt{\frac{1}{M} \sum_{m=1}^{M}\left[\frac{1}{T} \sum_{k=1}^{T}\left(\mathbf{x}_{k}^{m}-\hat{\mathbf{x}}_{k}^{m}\right)^{2}\right]},
\end{gathered}
$$




$$
r m s e^{\prime}=\sqrt{\frac{1}{M} \sum_{m=1}^{M}\left(\mathbf{x}_{k}^{m}-\hat{\mathbf{x}}_{k}^{m}\right)^{2}},
$$

where the mean of rmse is $\overline{r m s e}, r m s e^{\prime}$ is the rmse for $M$ simulations, $\mathbf{x}_{k}^{m}$ the real state at time $k$ for the $m$ th simulation, and $\hat{\mathbf{x}}_{k}^{m}$ the estimated state.

The mean estimation rmse values of the generic PF, LPF, and GAePF for 200 simulations are presented in Figures 2 and 3, respectively. As can be seen from the figures, the rmse of the LPF is lower than that of the other two algorithms. The root mean square error ( rmse) results of the three algorithms with $N=100$ and 200 are compared in Table 1 . It can be seen that the estimation accuracy of the LPF with just 100 particles is better than that of the other two filters with 200 particles. In other words, the LPF not only improves the accuracy, but also has a higher utilization rate, while its runtime is close to that of the GAePF.

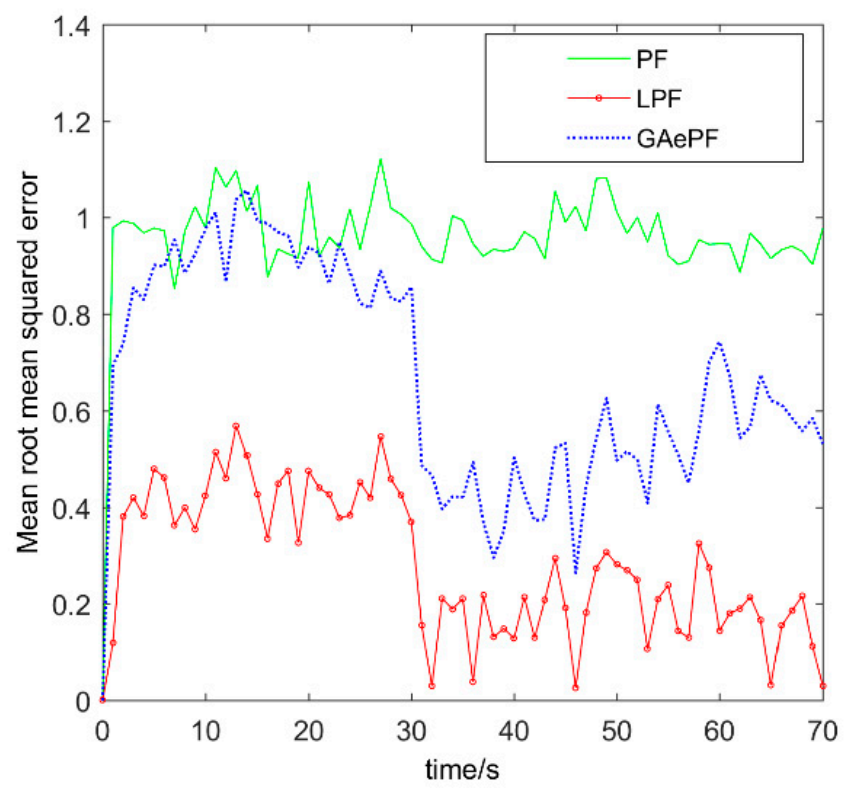

Figure 2. Mean root mean square error (rmse) with observation times for 200 simulations of three algorithms compared.

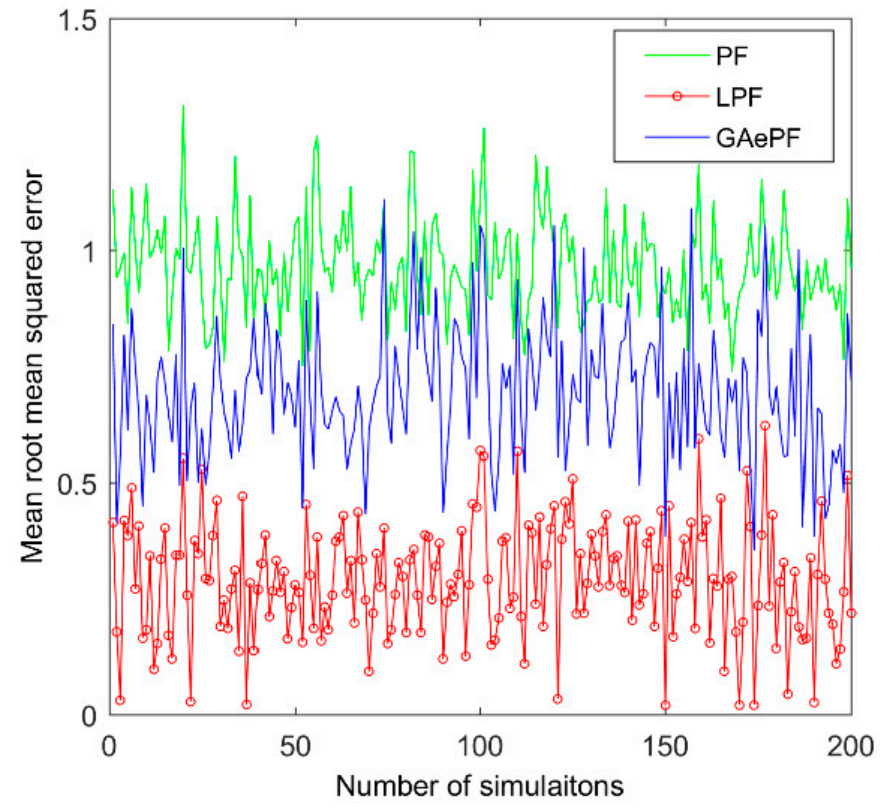

Figure 3. Mean rmse with simulation numbers of three algorithms compared. 
Table 1. Mean Root Mean Squared Error and Stop Time.

\begin{tabular}{cccc}
\hline \multirow{2}{*}{ Algorithm } & \multicolumn{3}{c}{ Performance Metrics } \\
\cline { 2 - 4 } & Mean Root Mean Squared Error & Stop Time & \multirow{2}{*}{$\boldsymbol{N}$} \\
\hline PF & 0.9604 & 0.2542 & \multirow{2}{*}{100} \\
GAePF & 0.8019 & 1.5454 & \\
\hline PF & 0.7604 & 0.6692 & \multirow{2}{*}{200} \\
GAePF & 0.6960 & 3.1651 & \\
\hline LPF & 0.2902 & 2.1539 & 100 \\
\hline
\end{tabular}

\subsection{RUL Prediction for Lithium-Ion Battery}

\subsubsection{Experimental Battery Datasets}

In this subsection, the proposed LPF framework for RUL prediction is validated against lithium-ion battery data provided by the NASA Ames Research Center Prognostics Center of Excellence [36]. The data were obtained at room temperature $\left(24^{\circ} \mathrm{C}\right)$.

The dataset contains four cells, labeled as B05, B06, B07, and B18. Their nominal capacity is $2000 \mathrm{mAh}$, while the nominal voltage is $3.7 \mathrm{~V}$. To estimate accelerated aging, multiple charge-discharge cycles were undertaken. All of the test cells went through the same constant-current (CC) and constant-voltage $(\mathrm{CV})$ charging with current maintained at $1.5 \mathrm{~A}$ until the voltage reaches the upper limit of $4.2 \mathrm{~V}$ and then maintained until the current dropped to $20 \mathrm{~mA}$. Similarly, discharging went through a constant current of $2 \mathrm{~A}$ until the voltages fell to $2.7 \mathrm{~V}, 2.5 \mathrm{~V}, 2.2 \mathrm{~V}$, and $2.5 \mathrm{~V}$ for battery B05, B06, B07, and B18, respectively. The failure threshold was $1400 \mathrm{mAh}$.

The capacity degradation trend is shown in Figure 4, where the degradation data were drawn using MATLAB ${ }^{\circledR \circledR}$ (MathWorks, Natick, MA, USA) curve fitting. It can be seen that the empirical model of Equation (4) fit the regression process. Figure 5 shows that if the parameters are accurately estimated, the model fits the degradation trend.

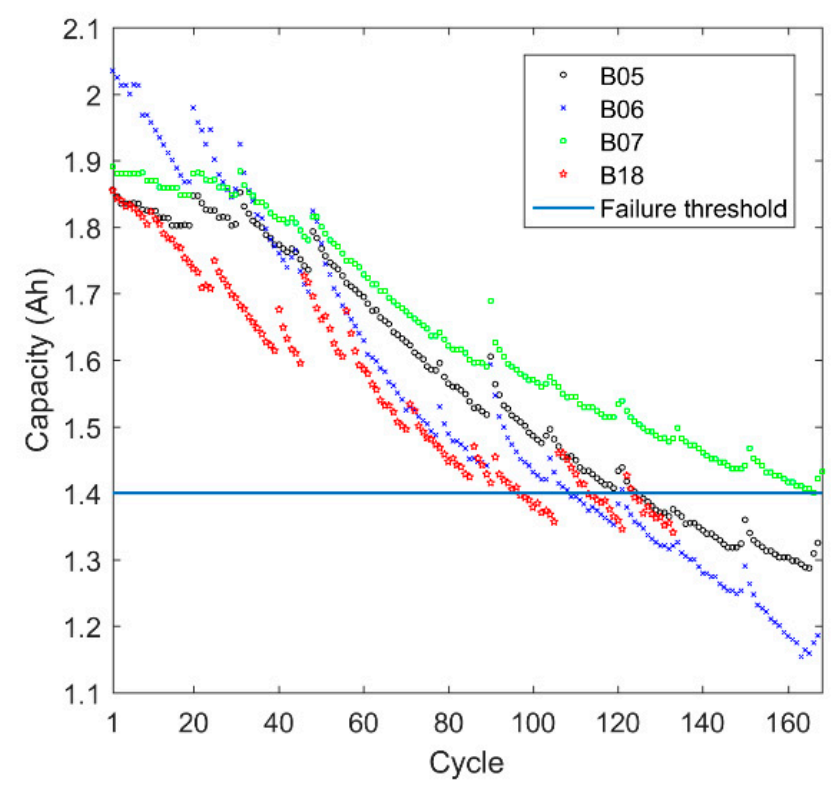

Figure 4. NASA Ames Research Center Prognostics Center of Excellence battery dataset.

To test the prediction accuracy, the data of batteries labeled B05, B06 and B07 were used as the training data. For validation, battery B18 was predicted using the LPF. For simplicity in this experiment, $\mu$ was set to 0.6 and $\sigma_{a}=1 \times 10^{-4}, \sigma_{b}=1 \times 10^{-6}, \sigma_{c}=1 \times 10^{-6}, \sigma_{d}=1 \times 10^{-7}$, and $\sigma_{n}=1 \times 10^{-3}$ for the Gaussian noise mode. Hence, only the four parameters $a, b, c$, and $d$ must be estimated in a particle. 
The parameters of the three known batteries were produced by the fitting to obtain initial training data, as shown in Table 2.

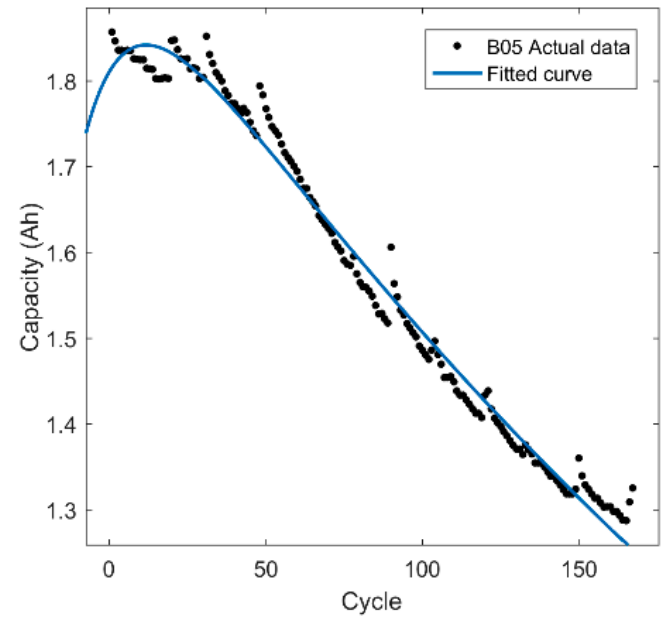

(a)

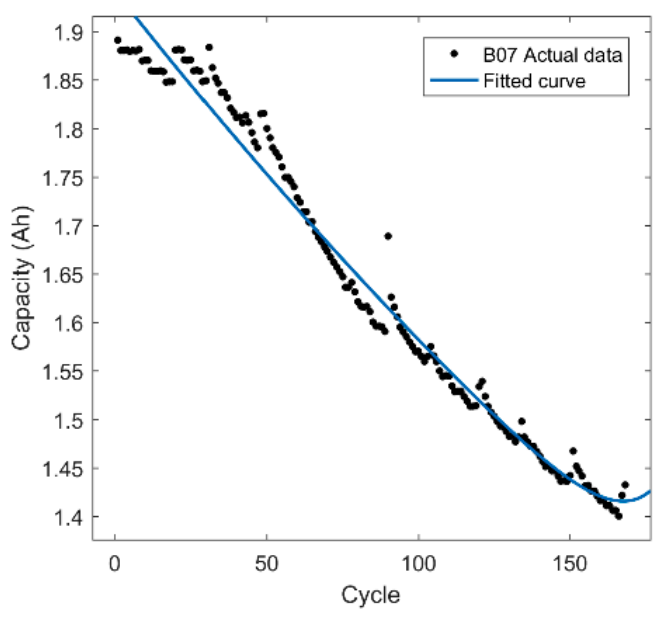

(c)

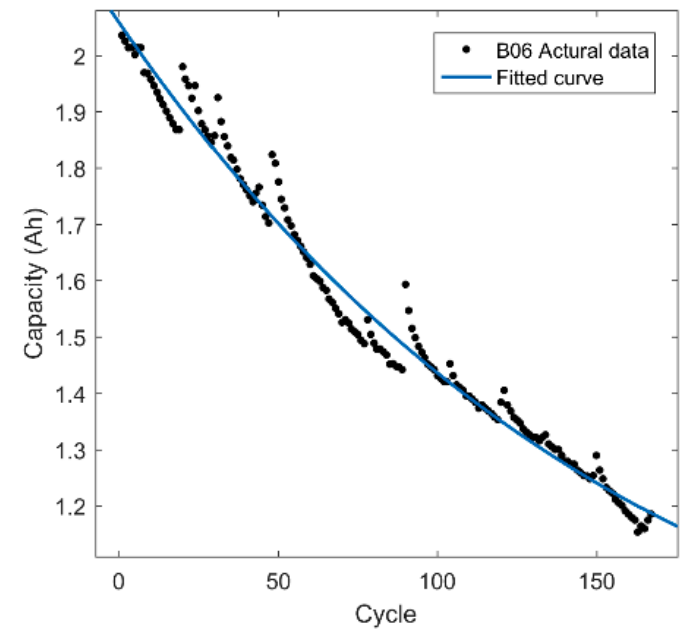

(b)

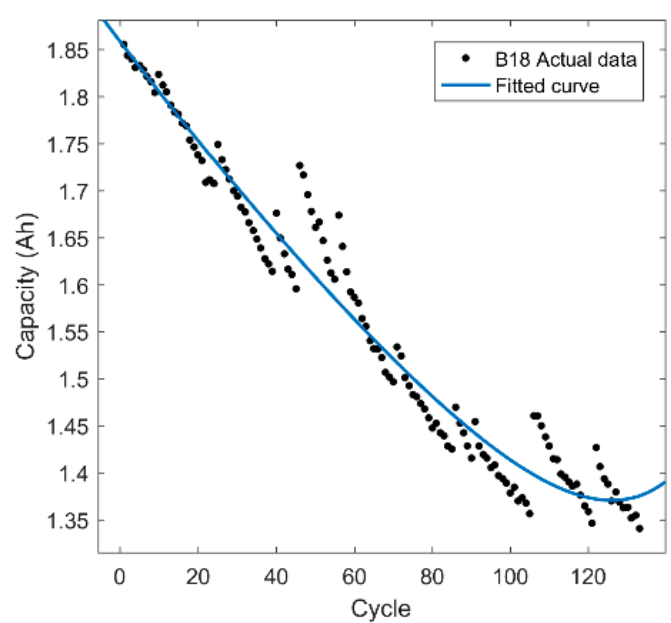

(d)

Figure 5. Degradation data and fitting curves of the four batteries: (a) B05; (b) B06; (c) B07; (d) B18.

Table 2. Model Parameters of Batteries and State Initialization.

\begin{tabular}{cccc}
\hline Battery ID & State Variables & Initial Value & Fitting Range \\
\hline \multirow{3}{*}{ B05 } & $a$ & 1.983 & $(1.963,2.004)$ \\
& $b$ & -0.002746 & $(-0.002838,-0.002654)$ \\
& $c$ & -0.1738 & $(-0.2006,-0.1469)$ \\
& $d$ & -0.06751 & $(-0.09014,-0.04488)$ \\
B06 & $a$ & 1.579 & $(-1.745,4.903)$ \\
& $b$ & -0.00549 & $(-0.01438,0.003402)$ \\
& $c$ & 0.4797 & $(-2.859,3.819)$ \\
B07 & $d$ & 0.0008834 & $(-0.01656,0.01833)$ \\
& $a$ & 1.942 & $(1.933,1.951)$ \\
& $b$ & -0.002052 & $(-0.002126,-0.001978)$ \\
& $c$ & $1.572 \times 10^{-7}$ & $\left(-2.198 \times 10^{-6}, 2.513 \times 10^{-6}\right)$ \\
Mean & $d$ & 0.07406 & - \\
& $a$ & 1.8347 & - \\
& $b$ & -0.003429 & - \\
\hline & $c$ & 0.101967 & - \\
& $d$ & 0.0024778 & \\
& & & $-1643)$ \\
& & & \\
& & &
\end{tabular}




\subsubsection{Results and Analysis}

In this work, the initial parameters were set as their corresponding mean values of batteries B05, B06, and B07 shown in Table 2. The number of particles $N$ was set to 100. To test the robustness of the LPF method, different numbers of training cycles were used to compare the prediction results, which were the first 33 and 70 measurement cycles. To test the relative prediction performance, the results were compared with those obtained using the standard PF. Furthermore, the model accuracy was evaluated on the RUL absolute error (AE), the RUL relative prediction error (RPE), and the capacity root-mean-square-error (RMSE). These are defined as:

$$
\begin{gathered}
\mathrm{AE}=\left|\mathrm{RUL} \mathrm{L}_{\text {true }}-\mathrm{RUL}_{\text {prediction }}\right|, \\
\mathrm{RPE}=\mathrm{AE} / \mathrm{RUL}_{\text {true }}, \\
R M S E_{\text {estimate }}=\sqrt{\frac{1}{C} \sum_{k=1}^{c}\left(Q_{k}-\hat{Q}_{k}\right),} \\
\operatorname{RMSE}_{\text {prediction }}=\sqrt{\frac{1}{C p} \sum_{k=\text { start }}^{c}\left(Q_{k}-\hat{Q}_{k}\right)},
\end{gathered}
$$

where $C$ is the number of measurement cycles, $Q_{k}$ is the estimate at cycle $k, C p$ is the number of cycles that must be predicted, and $\hat{Q}_{k}$ the prediction result at cycle $\mathrm{k}$.

\section{Prediction Performance Analysis}

Figure 6 shows the RUL prediction results from the standard PF and the proposed LPF, where the first 33 cycles were used to update the prediction. Compared with the dataset, it is apparent that the LPF offers better predictions.

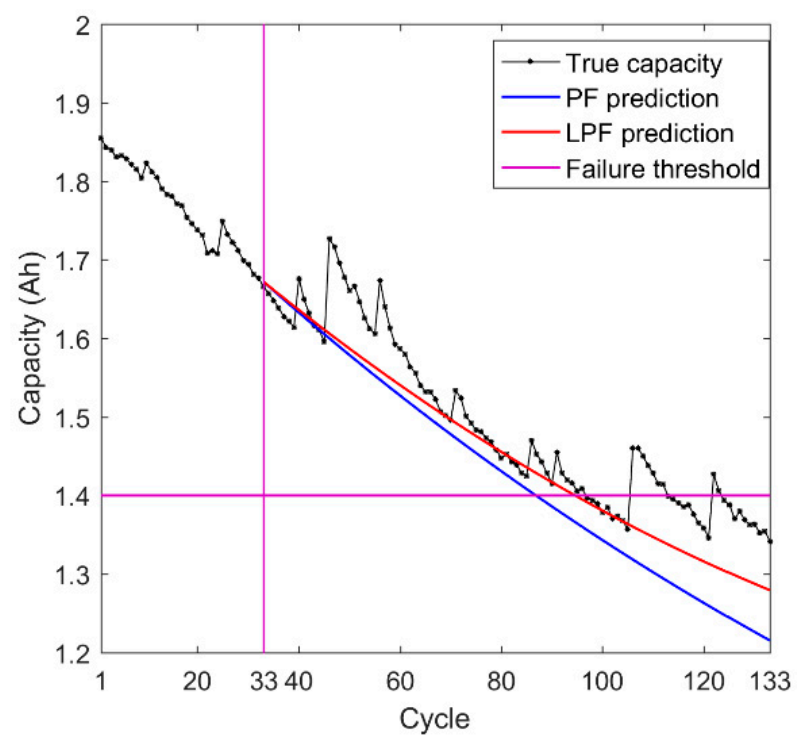

Figure 6. Prediction comparison using 33 cycles for battery B18 of a remaining useful life (RUL) of 97 cycles.

Figure 7 compares the histograms of the RULs predicted by the PF and LPF. It can be seen from Figure 7 a that, using the PF method, more than $10 \%$ of the particles failed to predict the RUL from existing measurements during the first 33 cycles, and the estimated predicted RULs of cycles were within the range [50,130], giving an average at 80 cycles, far away from the RUL data at 97 cycles. In contrast, Figure $7 \mathrm{~b}$ plots the performance of the LPF predicted RULs within the range [94, 100], showing $90 \%$ of the particles predicting 95 cycles, which were very close to the true RUL of 97 . 


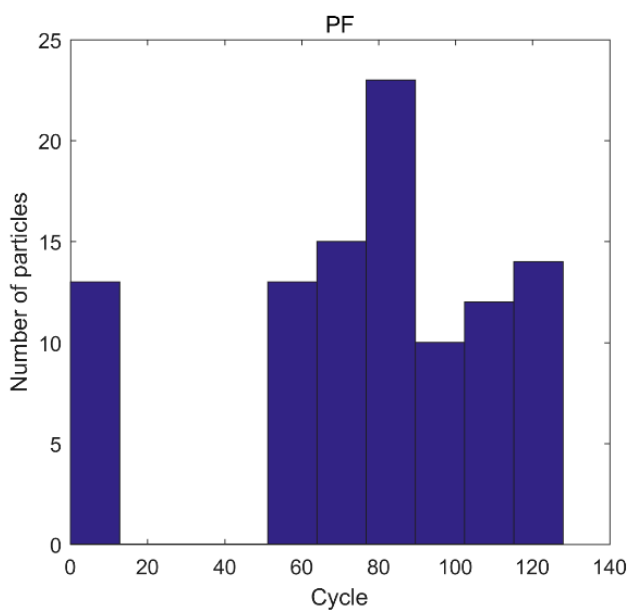

(a)

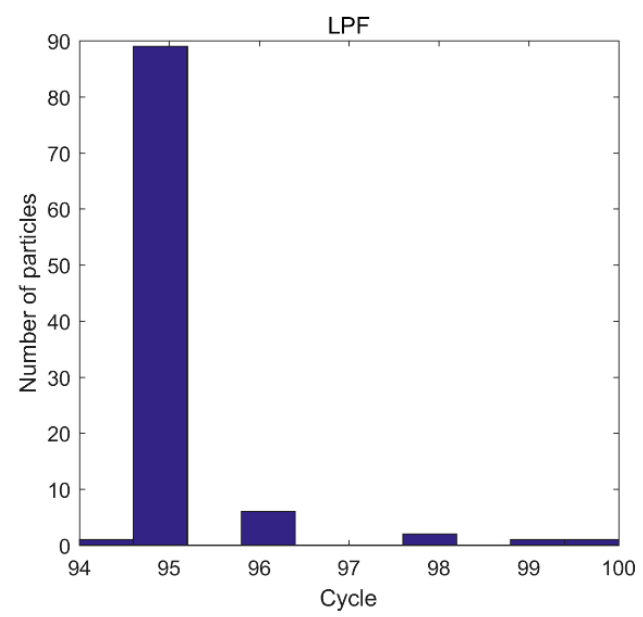

(b)

Figure 7. Histograms of predicted RUL distribution for battery B18, using 100 particles and 33 observations for the particle filter (PF) and the LPF methods: (a) PF; (b) LPF.

When using the first 70 cycles as measurement data to predict the RULs, Figure 8 similarly shows that the LPF offers better results. Figure 9a,b show the histograms of the RULs predicted by the PF and LPF, respectively. The same conclusions as drawn from Figures 6 and 7 can be drawn from Figure 9, confirming the robustness of the LPF method.

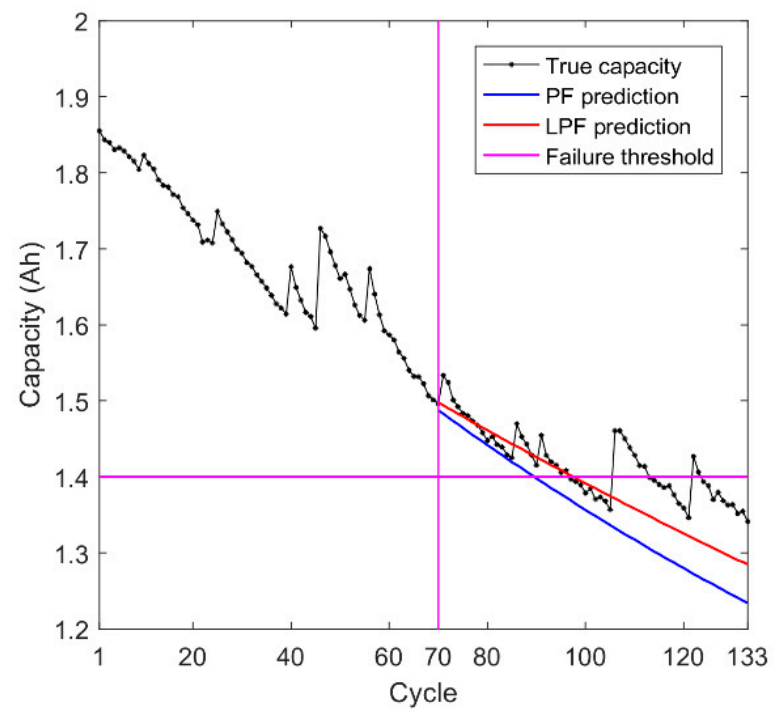

Figure 8. Prediction comparison using 70 cycles for battery B18 of a RUL of 97 cycles.

Figure 10a,b show the capacity RMSEs for the predictions using the PF and LPF methods, in which the first 33 and 70 cycles were used for the prediction, respectively. This confirms that the proposed LPF method results in a smaller RMSE than the PF.

Table 3 shows prediction errors between the two methods. It can be seen from the table that the predicted AEs, RPEs, and RMSEs obtained by the LPF are all smaller than those obtained with a standard PF. 


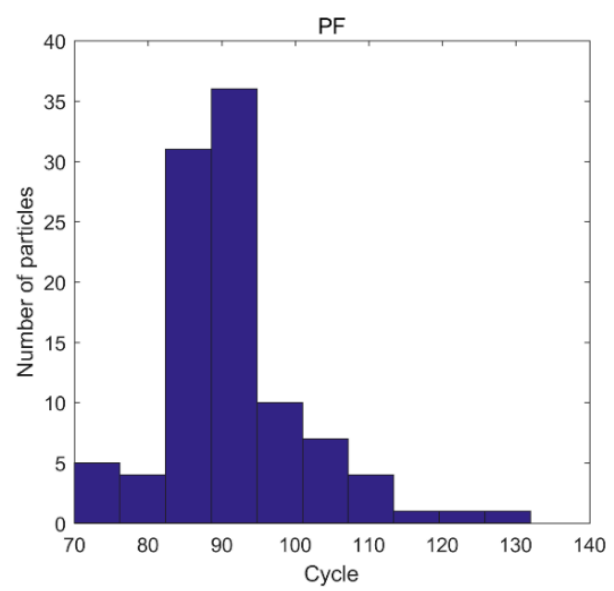

(a)

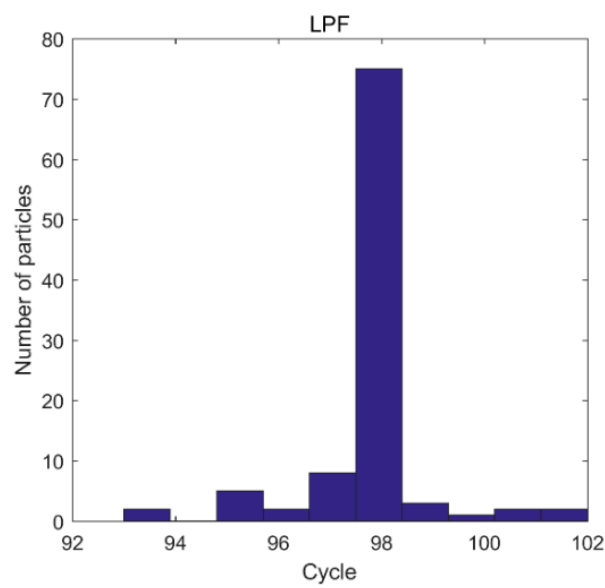

(b)

Figure 9. Histograms of predicted RUL distribution for battery B18, using 100 particles and 70 observations for the PF and the LPF methods: (a) PF; (b) LPF.

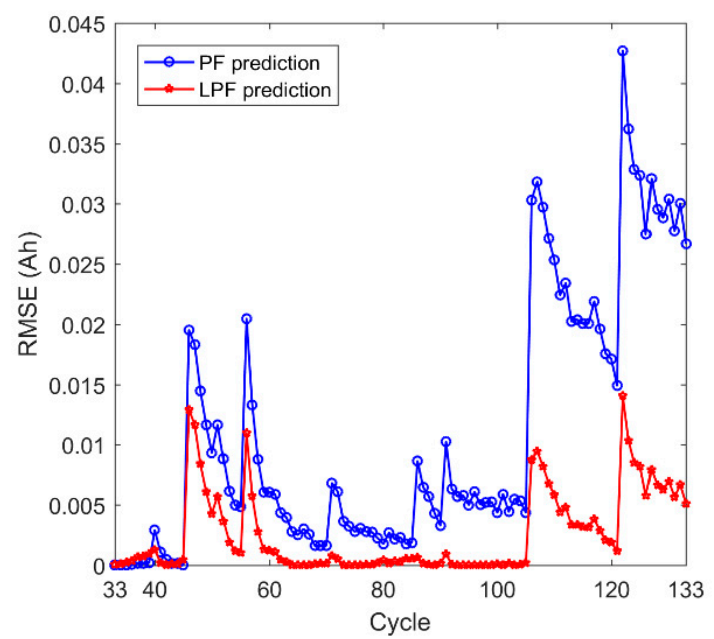

(a)

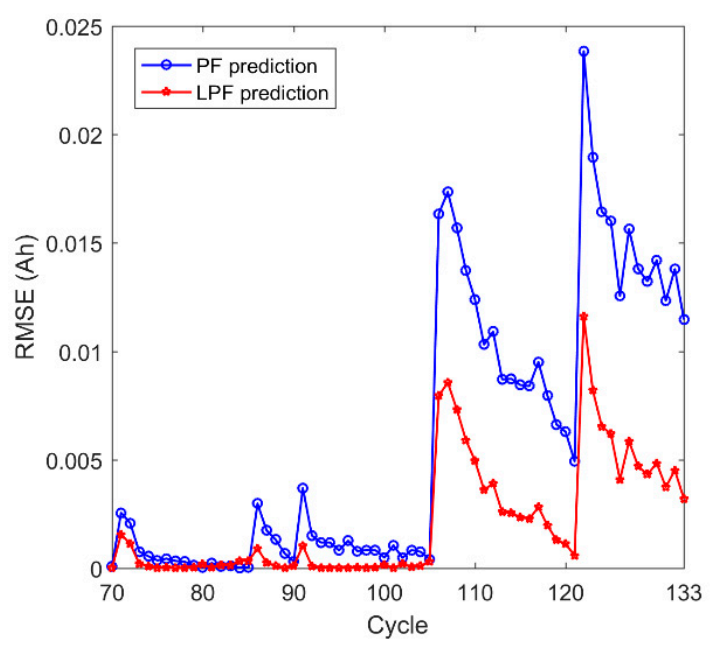

(b)

Figure 10. Capacity RMSEs for PF and LPF predictions: (a) Using 33 cycles as measurements; (b) Using 70 cycles as measurements.

Table 3. Prediction Results for Battery B18.

\begin{tabular}{ccccccc}
\hline Cycles & True RUL & Algorithms & Prediction RUL & AE & RPE & RMSE \\
\hline \multirow{2}{*}{33} & & PF & 87 & 10 & $10.3 \%$ & 0.09084 \\
& \multirow{3}{*}{70} & LPF & 95 & 2 & $2.1 \%$ & 0.04408 \\
& & PF & 90 & 7 & $7.2 \%$ & 0.07705 \\
& & LPF & 98 & 1 & $1 \%$ & 0.04597 \\
\hline
\end{tabular}

One-Step Ahead Prediction Analysis

As all of the measurements of the battery could not be obtained while it was working, one-step prediction performance will also be considered as an on-line prediction reference. Figure $11 \mathrm{a}, \mathrm{b}$ show the one-step-ahead capacity estimates using the standard PF and proposed LPF, respectively. Again, the LPF offers better results than the PF. To visualize the performance difference more clearly, the capacity RMSEs of the estimates at every cycle are compared in Figure 12 and mean RMSEs of all cycles are compared in Table 4. The results of these comparisons confirm that the LPF is a better method. 


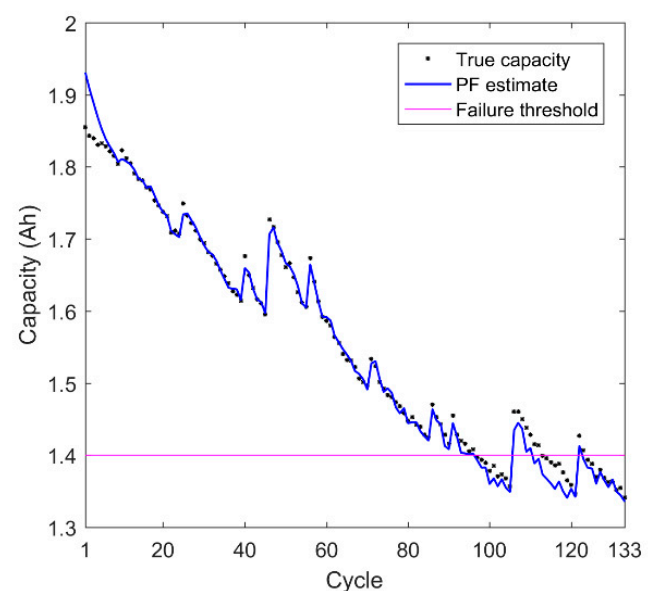

(a)

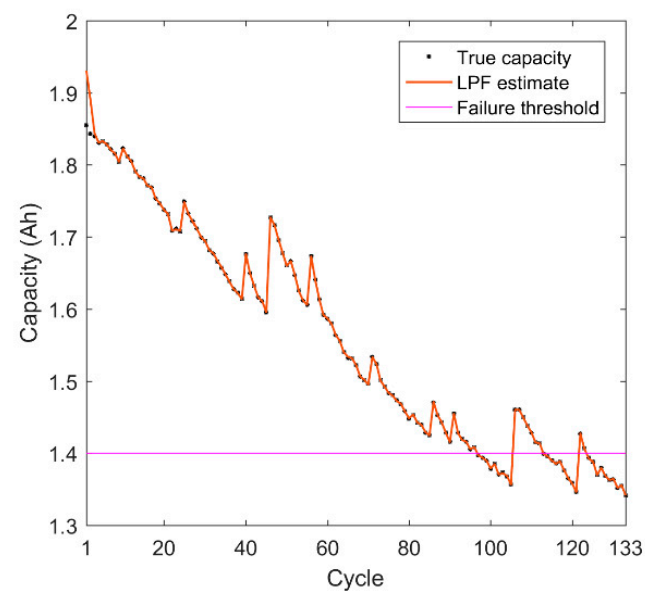

(b)

Figure 11. One-step-ahead estimates of capacity degradation trends for battery B18: (a) PF; (b) LPF.

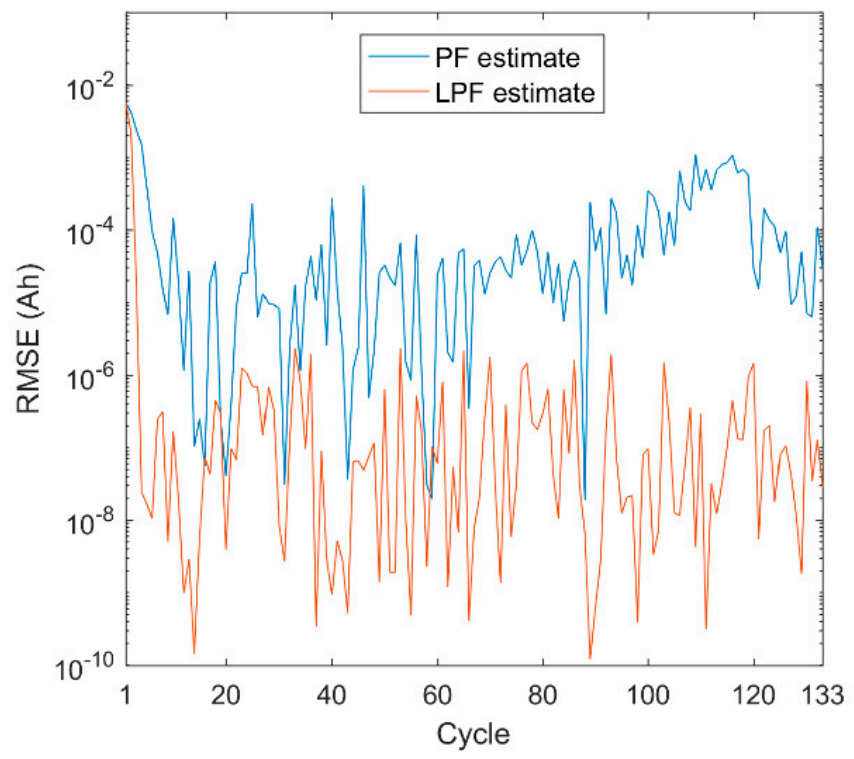

Figure 12. Comparison of capacity estimate RMSEs for battery B18 between the PF and the LPF methods.

Table 4. Mean RMSE of One-Step-Ahead Capacity Estimates.

\begin{tabular}{cc}
\hline Algorithms & Mean RMSE (Ah) \\
\hline PF & 0.01098 \\
LPF & 0.00706 \\
\hline
\end{tabular}

\section{Conclusions}

In this work, a novel approach to predicting lithium-ion battery's remaining useful life has been developed and evaluated. In the literature, effective forecast of remaining useful life has been reported using a particle filter, but it suffers from particle degeneracy and impoverishment deficiencies. The Lamarckian particle filter developed in this paper was to solve these problems for the first time to improve and broaden prediction of lithium-ion battery RUL.

Since the standard PF is a widely used algorithm for RUL prediction, we have used the RUL estimations from the traditional methods for comparison and have mapped a battery degradation model onto a particle filter problem. The LPF method developed in this paper is based on the PF estimates using the genetic algorithm framework with a Lamarckian inheritance mechanism. A Lamarckian inheritance operator has been developed to reduce particle degradation, enhance particle 
diversity and simplify the filtering process. Thus, the optimized particle will tend to cluster around the high likelihood region. The LPF has yielded better estimates of the RUL for cases considered with different numbers of measurement data, which are from NASA lithium-ion battery capacity degradation database, in terms of the average.

Moreover, according to histograms of the predicted RUL particle distribution using 33 and 70 observations, we draw the conclusion that more particles of the LPF when making a prediction were concentrated on the true RUL. In other words, the LPF well overcomes the lack of the particle collapsing problem, resulting in particle degeneracy and impoverishment. Further, better prediction results can be obtained using one-step ahead LPF prediction when the charge and discharge cycles proceed. This indicates that the proposed method has a good adaptability for lithium-ion battery degradation with non-linear and non-Gaussian characteristics.

It is expected that the LPF method can cope with more complex RUL models. Hence, in planned future research, the LPF method will be used to improve the underlying RUL model itself, and to make the prediction self-adaptive.

Author Contributions: L.L. designed the algorithm and drafted the manuscript. A.A.F.S. and Y.B. fed back on the algorithm and improved the manuscript. Y.L. coordinated the project and finalized the paper.

Funding: This research was funded in part by the Guangdong Higher Education Research Program under grant 2017KQNCX193, by Dongguan University of Technology under research grant 'Industry 4.0 Smart Design and Innovation Platform' (No. KCYKYQD2017014), and by the National Natural Science Foundation of China under Grant 71801044.

Conflicts of Interest: The authors declare no conflicts of interest. The funders had no role in the design of the study; in the collection, analyses, or interpretation of data; in the writing of the manuscript, or in the decision to publish the results.

\section{Nomenclature}

The following abbreviations are used in this manuscript.

$\begin{array}{ll}\text { AE } & \text { Absolute error } \\ \text { AI } & \text { Artificial intelligence } \\ \text { BMS } & \text { Battery management system } \\ \text { EKF } & \text { Extend Kalman filter } \\ \text { EOL } & \text { End of life } \\ \text { EV } & \text { Electric vehicle } \\ \text { GA } & \text { Genetic algorithm } \\ \text { GAePF } & \text { Elitism GA-based particle filter } \\ \text { LPF } & \text { Lamarckian particle filter } \\ \text { PF } & \text { Particle Filter } \\ \text { RMSE } & \text { Root mean square error } \\ \text { RPE } & \text { Relative prediction error } \\ \text { RUL } & \text { Remaining useful life } \\ \text { RVMs } & \text { Relevance vector machines } \\ \text { SD } & \text { Standard deviation } \\ \text { SOC } & \text { State of charging } \\ \text { SOH } & \text { State of health } \\ \text { SIR } & \text { Sequential Importance Resampling } \\ a, b, c, d & \text { Parameters of the double-exponential function } \\ k & \text { Number of charging cycles } \\ Q & \text { Remaining capacity of the battery } \\ Q_{k} & \text { Capacity of the battery at cycle index } k \\ N(\mu, \sigma) & \text { Gaussian noise with mean } \mu \text { and standard deviation }(\mathrm{SD}) \sigma \\ \mathbf{x}_{k} & \text { State variables at time } k\end{array}$




\begin{tabular}{ll}
$\mathbf{z}_{k}$ & Observations at time $k$ \\
$n_{\mathbf{x}}$ & Dimensions of $\mathbf{x}_{k}$ \\
$n_{\mathbf{z}}$ & Dimensions of $\mathbf{z}_{k}$ \\
$f(\cdot)$ & Known system function \\
$h(\cdot)$ & known Observation function \\
$\mathbf{u}_{k-1}$ & System noise \\
$\mathbf{v}_{k}$ & Observation noise \\
$\mathbf{x}_{0: k}=\left\{\mathbf{x}_{\left.0, \cdots, \mathbf{x}_{k}\right\}}\right\}$ & Sequences of the states \\
$\mathbf{z}_{1: k}=\left\{\mathbf{z}_{\left.1, \cdots, \mathbf{z}_{k}\right\}}\right.$ & Sequences of the observations \\
$p\left(\mathbf{x}_{0}\right)$ & Known a priori \\
$N$ & Number of particles \\
$\mathbf{x}_{k}^{i}(i=1, \cdots, N)$ & Set of particles at time $k$ \\
$\left\{w_{k}^{i}\right\}_{i=1}^{N}$ & Weight vector \\
$p\left(\mathbf{x}_{0: k} \mid \mathbf{z}_{1: k}\right)$ & Posterior probability density \\
$\mathbf{z}_{1: k}$ & All available measurements \\
$\delta(\cdot)$ & Dirac delta function \\
$p_{t}$ & Lamarckian probability \\
$n_{\mathrm{t}}$ & Numbers of genes transferred \\
$Q_{k}^{i}$ & Estimated capacity for each particle at cycle $k$ \\
$Q_{k+L}^{i}$ & L-step-ahead prediction at cycle $k$ \\
$L_{\mathbf{k}}^{\mathrm{i}}$ & ith trajectory is obtained \\
$Q_{r a t e d}$ & Rated capacity \\
$\rho$ & Inheritance probability \\
$C$ & Number of measurement cycles \\
$C p$ & Number of cycles that must be predicted \\
& \\
\hline &
\end{tabular}

\section{References}

1. Yu, Z.; Xiao, L.; Li, H.; Zhu, X.; Huai, R. Model parameter identification for lithium batteries using the coevolutionary particle swarm optimization method. IEEE Trans. Ind. Electron. 2017, 64, 5690-5700. [CrossRef]

2. Dong, G.; Wei, J.; Chen, Z. Kalman filter for onboard state of charge estimation and peak power capability analysis of lithium-ion batteries. J. Power Sources 2016, 328, 615-626. [CrossRef]

3. Kim, I.L.S. A technique for estimating the state of health of lithium batteries through a dual-sliding-mode observer. IEEE Trans. Power Electron. 2010, 25, 1013-1022.

4. Wu, J.; Wang, Y.; Zhang, X.; Chen, Z. A novel state of health estimation method of Li-ion battery using group method of data handling. J. Power Sources 2016, 327, 457-464. [CrossRef]

5. Xiong, R.; Yu, Q.; Shen, W.; Lin, C.; Sun, F. A sensor fault diagnosis method for a lithium-ion battery pack in electric vehicles. IEEE Trans. Power Electron. 2019, 34, 9709-9718. [CrossRef]

6. Barrera, J.; Galeano, N.; Maldonado, H. SoC estimation for lithium-ion batteries: Review and future challenges. Electronics 2017, 6, 102. [CrossRef]

7. Dong, G.; Chen, Z.; Wei, J.; Ling, Q. Battery health prognosis using Brownian motion modeling and particle filtering. IEEE Trans. Ind. Electron. 2018, 65, 8646-8655. [CrossRef]

8. Mejdoubi, A.; Oukaour, A.; Chaoi, H.; Gualous, H.; Sabor, J.; Slamani, Y. State-of-charge and state-of-health lithium-ion batteries diagnosis according to surface temperature variation. IEEE Trans. Ind. Electron. 2016, 63, 2391-2402. [CrossRef]

9. Eddahech, A.; Briat, O.; Bertrand, N.; Delétage, J.-Y.; Vinassa, J.-M. Behavior and state-of-health monitoring of Li-ion batteries using impedance spectroscopy and recurrent neural networks. Int. J. Elect. Power Energy Syst. 2012, 42, 487-494. [CrossRef]

10. Liu, D.; Zhou, J.; Pan, D.; Peng, Y.; Peng, X. Lithium-ion battery remaining useful life estimation with an optimized relevance vector machine algorithm with incremental learning. Measurement 2015, 63, 143-151. [CrossRef] 
11. Liu, D.; Yin, X.; Song, Y.; Liu, W.; Peng, Y. An on-line state of health estimation of lithium-ion battery using unscented particle filter. IEEE Access 2018, 6, 40990-41001. [CrossRef]

12. Kim, J.; Cho, B.-H. State-of-charge estimation and state-of-health prediction of a li-ion degraded battery based on an EKF combined with a per-unit system. IEEE Trans. Veh. Technol. 2011, 60, 4249-4260. [CrossRef]

13. Chen, Z.P.; Wang, Q.T. The application of UKF algorithm for 18650-type lithium battery SOH estimation. Appl. Mech. Mater. 2014, 519-520, 1079-1084. [CrossRef]

14. Hu, C.; Youn, B.D.; Chung, J. A multiscale framework with extended Kalman filter for lithium-ion battery SOC and capacity estimation. Appl. Energy 2012, 92, 694-704. [CrossRef]

15. Zheng, X.; Fang, H. An integrated unscented kalman filter and relevance vector regression approach for lithium-ion battery remaining useful life and short-term capacity prediction. Reliab. Eng. Syst. Saf. 2015, 144, 74-82. [CrossRef]

16. Liu, S.-L.; Cui, N.-X.; Zhang, C.-H. State of health estimation of lithium-ion battery based on AUKF. Power Electron. 2017, 11, 122-124.

17. Banerjee, A.; Burlina, P. Efficient particle filtering via sparse kernel density estimation. IEEE Trans. Image Process. 2010, 19, 2480-2490. [CrossRef] [PubMed]

18. Gordon, N.; Salmond, D.; Smith, A. Novel approach to nonlinear/non-Gaussian Bayesian state estimation. IEEE Proc. F 1993, 140, 107-113. [CrossRef]

19. Dong, G.; Chen, Z.; Wei, J.; Zhang, C.; Wang, P. An online model-based method for state of energy estimation of lithium-ion batteries using dual filters. J. Power Sources 2016, 301, 277-286. [CrossRef]

20. Dalal, M.; Ma, J.; He, D. Lithium-ion battery life prognostic health management system using particle filtering framework. Proc. Mech. Eng. Part O J. Risk Reliab. 2011, 225, 81-90. [CrossRef]

21. Liu, J.; Wang, W.; Ma, F. A regularized auxiliary particle filtering approach for system state estimation and battery life prediction. Smart Mater. Struct. 2011, 20, 075021. [CrossRef]

22. Miao, Q.; Xie, L.; Cui, H.; Liang, W.; Pecht, M. Remaining useful life prediction of lithium-ion battery with unscented particle filter technique. Microelectron. Reliab. 2012, 53, 805-810. [CrossRef]

23. Sbarufatti, C.; Corbetta, M.; Giglio, M.; Cadini, F. Adaptive prognosis of lithium-ion batteries based on the combination of particle filters and radial basis function neural networks. J. Power Sources 2017, 344, 128-140. [CrossRef]

24. Wang, D.; Yang, F.; Tsui, K.L.; Zhou, Q.; Bae, S.J. Remaining useful life prediction of lithium-ion batteries based on spherical cubature particle filter. IEEE Trans. Instrum. Meas. 2016, 65, 1282-1291. [CrossRef]

25. Zhang, X.; Miao, Q.; Liu, Z. Remaining useful life prediction of lithium-ion battery using an improved UPF method based on MCMC. Microelectron. Reliab. 2017, 75, 288-295. [CrossRef]

26. Zou, G.H.; Jing, Z.L.; Hu, H.-T. Particle filter algorithm based on optimizing combination resampling. J. Shanghai Jiaotong Univ. 2016, 40, 1135-1139.

27. Zhang, H.; Miao, Q.; Zhang, X.; Liu, Z. An improved unscented particle filter approach for lithium-ion battery remaining useful life prediction. Microelectron. Reliab. 2018, 81, 288-298. [CrossRef]

28. Duong, P.; Raghavan, N. Heuristic Kalman optimized particle filter for remaining useful life prediction of lithium-ion battery. Microelectron. Reliab. 2018, 81, 232-243. [CrossRef]

29. Tan, K.C.; Li, Y. Grey-box model identification via evolutionary computing. Control Eng. Pract. 2002, 10, 673-684. [CrossRef]

30. He, W.; Williard, N.; Osterman, M.; Pecht, M. Prognostics of lithium-ion batteries based on Dempster-Shafer theory and the Bayesian Monte Carlo method. J. Power Sources 2011, 196, 10314-10321. [CrossRef]

31. Yin, S.; Zhu, X. Intelligent Particle Filter and Its Application to Fault Detection of Nonlinear System. IEEE Trans. Ind. Electron. 2015, 62, 3852-3861. [CrossRef]

32. Dawkins, R. The Blind Watchmaker: Why the Evidence of Evolution Reveals a Universe without Design; W.W. Norton \& Co Inc.: New York, NY, USA, 1996.

33. Brian, R. A Lamarckian Evolution Strategy for Genetic Algorithms. In The Practical Handbook of Genetic Algorithms; Chambers, L., Ed.; CRC Press: Boca Raton, FL, USA, 1999.

34. Tan, K.C.; Li, Y. Performance-based control system design automation via evolutionary computing. Eng. Appl. Artif. Intell. 2001, 14, 473-486. [CrossRef] 
35. Wang, Y.; Sun, F.; Zhang, Y. Central difference particle filter applied to transfer alignment for SINS on missiles. IEEE Trans. Aerosp. Electron. Syst. 2012, 48, 375-387. [CrossRef]

36. Saha, B.; Goebel, K. Battery Data Set. NASA Ames Prognostics Data Repository, NASA Ames Research Centre, Moffett Field, CA, USA. 2007. Available online: https://ti.arc.nasa.gov/tech/dash/groups/pcoe/prognosticdata-repository/ (accessed on 1 March 2019). 